\title{
Nanoemulsions as a Vehicle for Drugs and Cosmetics
}

\author{
Rocha-Filho PA*, Maruno M, Oliveira B, Bernardi DS, Gumiero VC, Pereira TA \\ Department of Pharmaceutical Sciences, University of Sao Paulo, Brazil
}

Received: Dec ember 12, 2013; Accepted: January 27, 2014; Published: January 29, 2014

"Corresponding author: Rocha-Filho PA, Department of Pharmaceutical Sciences, Faculty of Pharmaceutical Sciences of Ribeirao Preto, University of Sao Paulo, Av. do Café, s/nº - Bairro Monte Alegre - Ribeirão Preto - SP-CEP 14040-903, Brazil, Tel: 55-163-602-4214/3602-4279, E-mail: pedranjo@ fcfrp.usp.br

\section{Introduction}

Nowadays, pharmaceutical and cosmetic industries have had great interest in nanoemulsions due to their characteristics, such as high kinetic stability; skin delivery efficiency of active substance or drugs; excellent sensorial and esthetic aspects, besides the need for less surfactant (5-10\%) when compared to microemulsions, which reduces the possibility of skin irritation and production costs $[1,2]$.

Nanoemulsions are special systems with uniform and extremely small droplet size, in the range of $20-500 \mathrm{~nm}$. Due to their characteristic size, nanoemulsions can be optically transparent or translucent and have low viscosity that results in excellent spreadability and humectation. These benefits make them very interesting to be used in many applications, for example, in cosmetic and pharmaceutical field as personal care or skin care formulations and as drug delivery systems, respectively.

Different oils can be used in order to obtain nanoemulsions, like andiroba and copaiba oils. Both oils are widely used in cosmetics due to their emollient properties and compatibility with the lipids of the stratum corneum. Moreover, these oils have attractive properties to be used in skin care products. The andiroba oil (Carapa guianensis) and copaiba (Copaifera sp.) have been documented containing repellent action and may represent a safe alternative to synthetic repellents considering the risk of toxicity in certain cases. Andiroba oil is a natural product used by Amazonian Indians to ward off insects [3]. Several studies have identified the presence of limonoids in the seeds of Carapa, which presentinsecticidal and repellent activity [4]. Thus, it is interesting because thousands of people get sick and even are death victims each year, especially from mosquitoes that transmit diseases such as dengue and yellow fever. The mosquito-borne diseases affect more than 700 million people each year [5]. According to the World Health Organization, two fifths of the population is at risk of being infected with dengue virus, and more than 100 countries have been affected by epidemics of dengue and dengue hemorrhagic fever [6]. The use of repellents against Aedes aegypti is one of the strategies used to prevent and reduce the incidence of diseases transmitted by these arthropods [7].
Our nanoemulsion research is based on andiroba, copaiba and other vegetable oils, such as bran rice, green coffee, raspberry, sesame indicum, passion fruit, peach and urucum oils. Some additives like polyhydric umectants (glycerol, propylenglycol, sorbitol), lanolin derivatives (ethoxylated or acethylated), some specific cosmetic components (areca, licorice and portulaca extracts) and essential oil (lavender oil) have been used to obtain nanoemulsions.

The emulsification process can affect the droplet size, the distribution and the stability of nanoemulsions $[8,9]$. Nanoemulsions can be obtained by high energy emulsification methods, where a shear rate is promoted in order to deform the particle $[10,11,12]$ or by low energy emulsification methods, which explore the systems' physicochemical properties through the change in the spontaneous curvature of the surfactant with temperature variation (PIT-Phase Inversion Temperature) or the composition variation (PIC-Phase Inversion Composition) $[8,13,14]$. Another method employs dilution of formulation that presents cubic phase structures. These nanoemulsions show the same size (nm) in all dilution [15].

In order to study the process of nanoemulsion obtainment, the following parameters [16] may be evaluated: 1) effect of different surfactant quantity; 2) influence of the order of component addition: the surfactant, oil and aqueous phase, 3) influence of temperature emulsification; 4) effect of agitation speed; 5) influence of the surfactant amount; 6) determination of the phase inversion temperature- determination of Emulsion Phase Inversion (EPI).

However, some studies show that surfactant blends are more effective than isolated ones, since the blends disperse and solubilize more the internal phase within the continuous one, being therefore possible to employ a smaller ratio of surfactant and produce more long-term stable emulsions [14,17]. Some of the surfactant systems employed in our nanoemulsion research were based on castor oil derivatives with different molecules of ethylene oxide (15 to 50 ).

Different nanosystems obtained in our research were characterized according some parameters cited before and also evaluated for anti-inflammatory, antioxydant and insect repellent activity and/or cosmetic applications. 


\section{Some examples of nanoemulsions developed with vegetable oils and some evaluation performed in our laboratory}

\section{Nanoemulsions containing sesame indicum and raspberry oils}

Very stable nanoemulsions were obtained with sesame indicum and raspberry oil and non-ionic surfactants at HLB value 8.0 (Figure 1). These nanoemulsions showed stability for 90 days (Table 1).

In this study, with other data not shown, we could conclude that the surfactant type, composition concentration, addition order, temperature, speed and time of emulsification were critical factors in obtaining low polydispersity index of nanoemulsions.

Antioxidant activity: The nanoemulsions of sesame oil or sesame and raspberry oil presented antioxidant activity of $68.71 \%$ and $67.75 \%$, respectively [18]. (Data not published).

In vitro evaluation: The Hen's Egg Test-Chorioallantoic Membrane (HET- CAM) method was performed in order to evaluate irritancy of nanoemulsions from blend of sesame and raspberry oil. The results revealed that nanoemulsions showed slightly irritating power and can be used as cosmetics $[19,20]$.

\section{Nanoemulsions containing andiroba and copaiba oils}

Particle size: The diameter sizes of these nanoemulsions were also determined with Delsa light scattering 440DX. The sizes $(\mathrm{nm})$ of nanoemulsions with andiroba oil, copaiba oil and blend of oils (andiroba oil+ copaiba oil) were $236 \pm 50,130 \pm 47$ and $228 \pm 64$, respectively.

In vivo evaluation: The topical use of nanoemulsions containing andiroba and copaiba oils [19] displayed repellent activity compared to DEET (commercial product) (Figure 2).

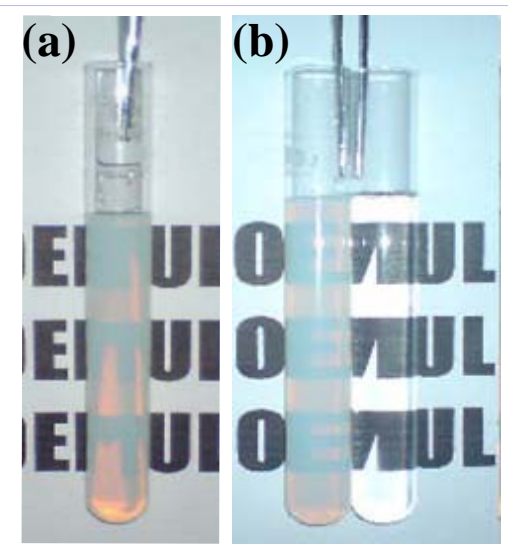

Figure 1: Nanoemulsion containing Sesame indicum and raspberry oils (a) compared with purified water (b). Particle size: The sesame indicum and raspberry nanoemulsions had their droplet diameter size determined. The diameter sizes were determined with Delsa light scattering 440DX. The nanoemulsions presented diameter size between 50 and $150 \mathrm{~nm}$. A mean diameter size of the nanoemulsions was $50 \mathrm{~nm}$ (Table 1).
Table 1: Droplet diameter size (nm) and polydispersity index of sesame nanoemulsions prepared at different temperature as function of time: 7 , 15 and 90 days. $\omega=$ polidispersity index.

\begin{tabular}{|c|c|c|c|c|}
\hline \multirow{2}{*}{ Temperature $\left( \pm 1^{\circ} \mathrm{C}\right) \downarrow$} & \multicolumn{3}{|c|}{ Time (days) } & \multirow{2}{*}{$\left(\begin{array}{c}\omega \\
\left(x 10^{-22} \mathrm{~m}^{3} \mathrm{~s}^{-1}\right)\end{array}\right.$} \\
\hline & 7 & 15 & 90 & \\
\hline 35 & $17 \pm 2.0$ & $18 \pm 3.3$ & $52 \pm 4.8$ & 0.8481 \\
\hline 45 & $17 \pm 1.4$ & $21 \pm 2.7$ & $33 \pm 5.5$ & 0.1939 \\
\hline 55 & $17 \pm 1.4$ & $16 \pm 1.9$ & $54 \pm 4.4$ & 0.9534 \\
\hline 65 & $17 \pm 1.4$ & $17 \pm 1.4$ & $46 \pm 9$ & 0.5776 \\
\hline 75 & $17 \pm 1.4$ & $17 \pm 1.4$ & $48 \pm 7.6$ & 0.6605 \\
\hline 85 & $15 \pm 2.5$ & $17 \pm 1.4$ & $61 \pm 11$ & 1.3975 \\
\hline 90 & $16 \pm 1.9$ & $17 \pm 1.4$ & $31 \pm 3.8$ & 0.1605 \\
\hline
\end{tabular}

The test repellent revealed that the nanoemulsions composed of andiroba oil and blend of oils (andiroba oil + copaiba oil) are able to repel mosquitoes Aedes aegypti for a period of 30 minutes. These results are statistically significant in the control group.

\section{Nanoemulsions containing rice bran seed oil}

In vitro evaluation: One of the evaluations performed in nanoemulsions containing rice bran seed oil was HET- CAM method. According this method, the nanoemulsions are also suitable to be used as cosmetics, since they presented slightly irritating power $[19,20]$.

In vivo evaluation: The nanoemulsions containing rice bran seed oil were evaluated by topical use. When they applied to the skin of volunteers, the nanoemulsion increased the relative hydration and the oiliness of skin and maintained normal $\mathrm{pH}$ values of skin. These nanoemulsions could serve as an alternative treatment for skin diseases, such as atopic dermatitis and psoriasis [20].

\section{Some additives in different nanosystems}

Addition of areca, licorice and portulaca natural extracts in babassu nanoemulsion: The addition of these natural extracts $(3.0 \%)$ in babassu nanoemulsion influenced the antioxidant activity. The nanoemsulsion added of these natural extracts presented antioxidant activity of $34.19 \pm 1.03$ compared to the same emulsion added of BHT (41.87 \pm 0.77 ) (data not yet published). However, the addition did not influence the initial droplet size. These nanoemulsions showed high kinetic stability in 3 months (Figure 3). Moreover, the babassu nanoemulsions showed slightly irritating power according to HET- CAM method, being able to be used as cosmetic products $[19,20]$.

Addition of lavender essential oil in the nanosystem containing passion fruit oil: The addition of lavender essential oil $(2.0 \%)$ in this nanosystem caused a reduction in the droplet size comparing to the nanoemulsions composed with only passion fruit oil (Figure 4).

Addition of green coffee oil in urucum (bixa orellana) nanoemulsion: green coffee oil was added in urucum nanoemulsion in different concentrations (A- $0.0 \%, \mathrm{~B}-2.0 \%$ and C- 3.0\%) [21]. The nanoemulsions were assessed $24 \mathrm{hs}$ after the preparation and the results for $\mathrm{pH}$, conductivity $(\mu \mathrm{s} / \mathrm{cm})$ and mean droplet size $(\mathrm{nm})$ were, respectively: A- $6.2 \pm 0.1,89.0 \pm 0.5$, 


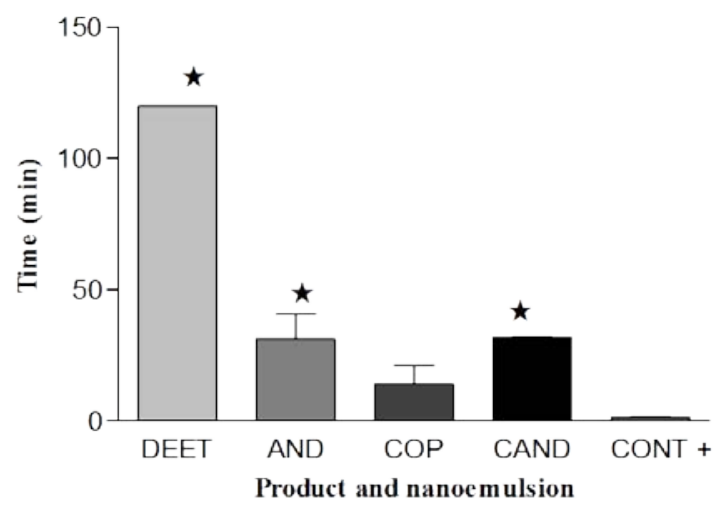

Figure 2: Repellent time (minutes) for commercial product and developed nanoemulsions containing vegetable oils (AND: andiroba oil; COP: copaiba oil; CAND: andiroba+ copaiba oil; CONT+: control; DEET: commercial product).

(A)

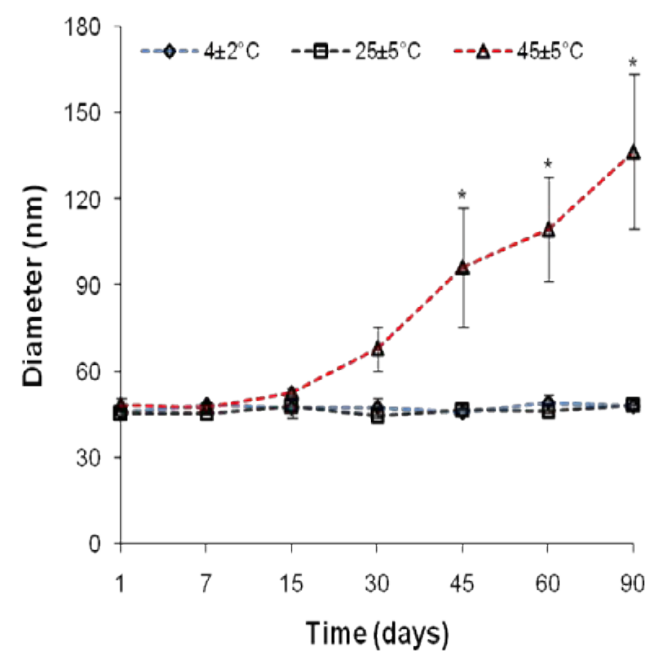

(B)

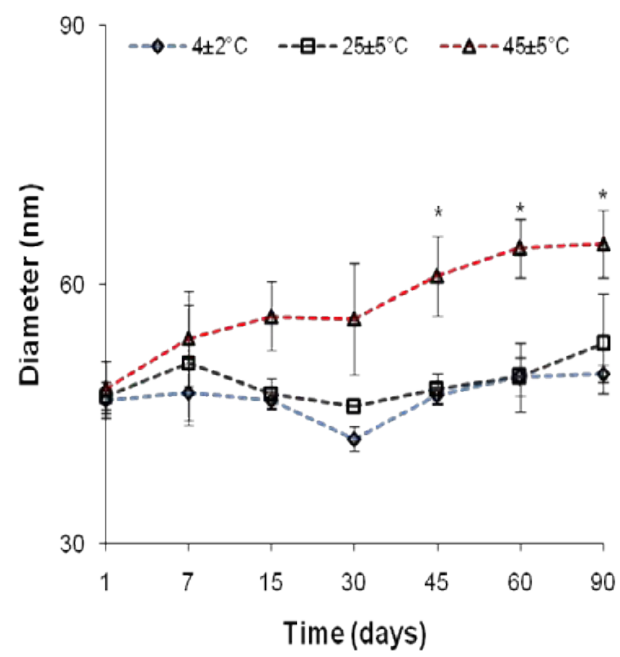

Figure 3: Nanoemulsion droplet size in function of storage temperature $\left(4 \pm 2,25 \pm 5,45 \pm 5^{\circ} \mathrm{C}\right)$ during 90 days. $(\mathrm{A})$ and (B) different formulations from diagram ternary.

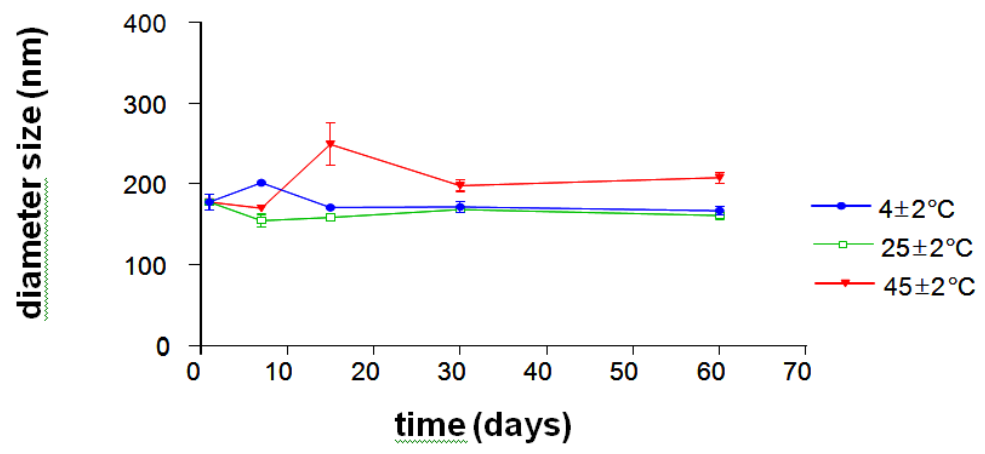

Figure 4: Nanoemulsion particle size obtained with passion fruit oil (5.0\%) and lavender essential oil (2.0\%) exposed at different conditions as time function. 
(A- EL)

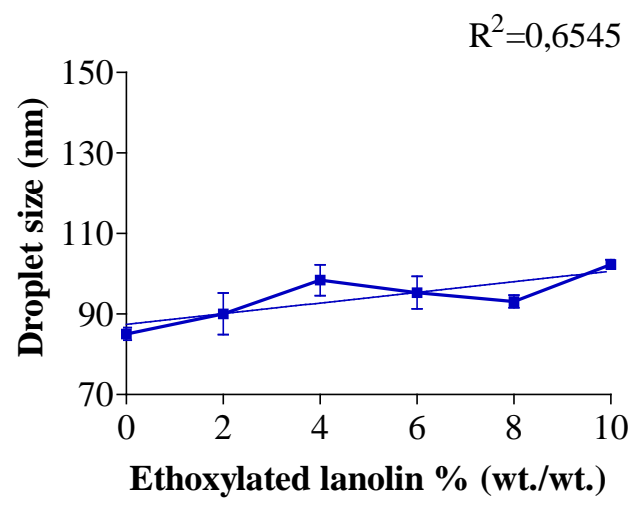

(B- AL)

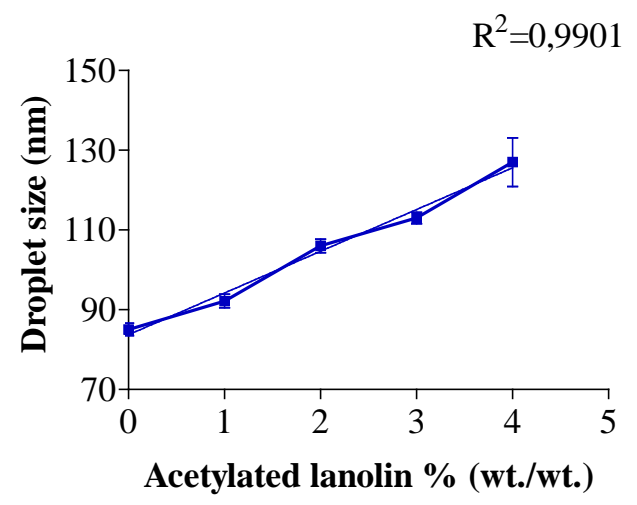

Figure 5: Droplet mean size of nanoemulsions as a function of lanolin derivatives concentration. (A) EL and (B) AL.

(A- EL)

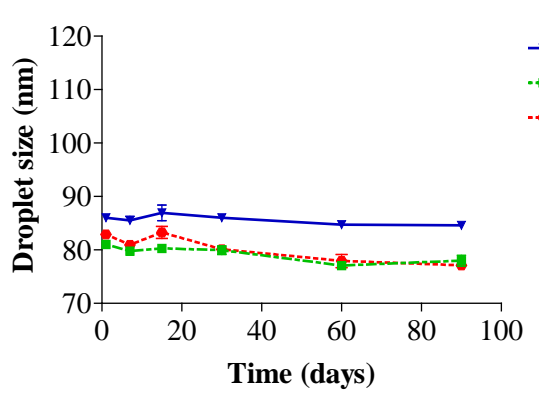

$(\mathrm{B}-\mathrm{AL})$

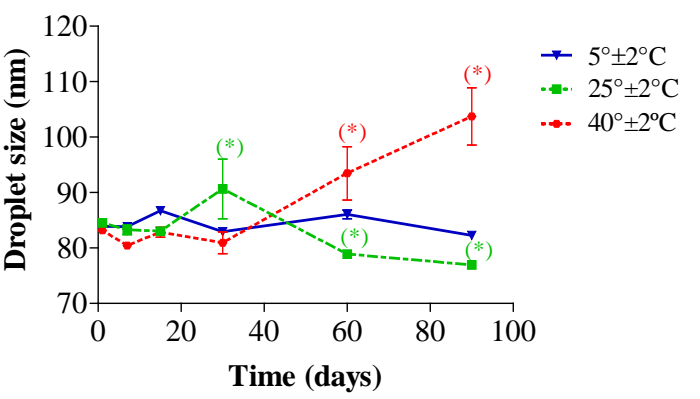

Figure 6: Droplet mean size of nanoemulsions added with lanolin derivatives submitted to different storage temperatures in function of time. (A) EL and (B) AL.

$90.0 \pm 10.7 ;$ B- $6.4 \pm 0.2, \quad 106.4 \pm 8.7,65.7 \pm 2.5$ and $C \quad 6.7 \pm 0.1$ $116.3 \pm 0.2,94.0 \pm 4.4$. They remained stable for 7 days and after thermal stress $\left(85^{\circ} \mathrm{C}\right)$ with no significant variation.

Addition of lanolin derivatives in nanoemulsions based on raspberry seed oil+ peach kernel oil+ passion fruit oil: the addition of lanolin derivatives, ethoxylated (EL) or acethylated (AL), in these nanoemulsions influenced droplet size and electrical conductivity of the systems, however, such changes did not compromise their stability, since the droplet size remained within the nanometer range (20-200nm) (Figure 5). Nanoemulsions added with EL were more stable than those with AL (Figure 6). Moreover, they are recommended to be employed as vehicle for pharmaceuticals and cosmetics.

\section{Conclusion}

According to this short communication

- It is possible to observe the importance of studying the parameters that can influence the nanoemulsion obtainment;

The composition of nanoemulsion, and mainly, the oily phase can provide attractive properties for the products;
- $\quad$ Nanoemulsions are an interesting and promising vehicle for drugs and cosmetics, as well as they hold wide development possibilities and numerous benefits in the pharmaceutical and cosmetic field.

\section{References}

1. Tadros T, Izquierdo P, Esquena J, Solans C (2004) Formation and stability of nano-emulsions. Adv Colloid Interface Sci 108-109: 303318.

2. Maruno M, Rocha-Filho PA (2009) O/W nanoemulsion after 15 years of preparation: a suitable vehicle for pharmaceutical and cosmetic applications. J Disp Sci Tech 31(1): 17-22.

3. Plowden C (2004) The ecology and harvest of andiroba seeds for oil production. Conservation \& Society, SAGE Publications, New Delhi/ Thousand Oaks/London.

4. Ambrozin ARP, Leite AC, Bueno FC, Vieira PC, Fernandes JB, et al. (2006) Limonoids from Andiroba Oil and Cedrela fissilis and their Insecticidal Activity. J Braz Chem Soc 17(3): 542-547.

5. Fradin MS, Day JF (2002) Comparative efficacy of insect repellents against mosquito bites. N Engl J Med 347(1): 13-18.

6. Cruz RR (2002) Strategies for controlling dengue and Aedes aegypti in the Americas. Rev Cubana Med Trop 54(3): 189-201. 
7. Thavara U, Tawatsin A, Chompoosri J, Suwonkerd W, Chansang UR, et al. (2001) Laboratory and field evaluations of the insect repellent 3535 (ethyl butylacetylaminopropionate) and deet against mosquito vectors in Thailand. J Am Mosq Control Assoc 17(3): 190-195.

8. Morais JM, Santos ODH, Delicato T, Gonçalves RA, Rocha Filho PA (2006) Physicochemical characterization of canola oil/ water nanoemulsions obtained by determination of required HLB number and emulsion phase inversion methods. Journal of Dispersion Science and Technology 27(1): 109-115.

9. Pey CM, Maestro A,SoléI, González C, Solans C, etal. (2006) Optimization of nano-emulsions prepared by low-energy emulsification methods at constant temperature using a factorial design study. Colloids and Surfaces A: Physicochemical and Engineering Aspects 288(1-3): 144150.

10. Usón N, Garcia MJ, Solans C (2004) Formation of water-in-oil (W/O) nano-emulsions in a water/mixed non-ionic surfactant/oil systems prepared by a low-energy emulsification method. Colloids and Surfaces A: Physicochemical and Engineering Aspects 250(1-3): 415421.

11. Tadros T, Izquierdo P, Esquena J, Solans C (2004) Formation and stability of nano-emultions. Adv Colloid Interface Sci 108-109: 303318.

12. Kourniatis LR, Spinelli L, Mansur CRE, González G (2010) Orange oil/water nanoemulsions prepared by high pressure homogenizer. Química Nova 33(2): 295-300.

13. Ee SL, Duan X, Liew J, Nguyen D (2008) Droplet size and stability of nano-emulsion produced by the temperature phase inversion method. Chemical Engineering Journal 140(1-3): 626-631.
14. Peng LC, Liu CH, Kwan CC, Huang KF (2010) Optimization of waterin-oil nanoemulsion by mixed surfactants. Colloids and Surfaces A: Physicochemical and Engineering Aspects 370(1-3): 136-142.

15. Pereira TA, Oliveira ECV, Guerreiro CM, Bortoloto J, Rocha-Filho PA (2010) Nanoemulsions attainment from a "ringig gel-Pm3n". $5^{\text {th }}$ World Congress on Emulsion, France.

16. Forgiarini A, Esquena J, Gonzalez C, Solans C (2001) Formation of nano-emulsions by low-energy emulsification methods at constant temperature. Langmuir 17(7): 2076-2083.

17. Porras M, Solans C, González C, Martínez A, Guinart A, et al. (2004) Studies of formation of W/O nano-emulsions. Colloids and Surfaces A: Physicochemical and Engineering Aspects 249(1-3): 115-118.

18. Maruno M, Rocha-Filho PA (2012) Characterization of nanoemulsions containing Sesame indicum oil and raspberry oil: antioxidant activity and toxicity (HET-CAM) evaluation. Vingt cinquièmes Entretiens du Centre Jacques Cartier, France.

19. Oliveira BR, Rocha-Filho PA (2012) Development and evaluation of nanoemulsions with Carapa guianensis and Copaifera sp. oil, and evaluation of repellency against Aedes aegypti. Vingt cinquièmes Entretiens du Centre Jacques Cartier, France.

20. Bernardi DS, Pereira TA, Maciel NR, Bortoloto J, Viera GS, et al. (2011) Formation and stability of oil-in-water nanoemulsions containing rice bran oil: in vitro and in vivo assessments. Journal of Nanobiotechnology 9: 44 .

21. Rocha-Filho PA, Agostinho LC, Gumiero VC (2010) Development and evaluation of nanoemulsions containing urucum oil added or not of green coffee oil. $5^{\text {th }}$ World Congress on Emulsion, France. 